REVISTA

EDUCACIÓN LAS

AMÉRICAS

FACULTAD DE EDUCACIÓN

UNIVERSIDAD DE LAS AMÉRICAS
Revista Educación las Américas

ISSN: 0719-7128

ccalisto@udla.cl

Universidad de Las Américas

Chile

Caracterización de prácticas educativas inclusivas en modalidad regular de educación para personas jóvenes y adultos. Un estudio de caso en el segundo nivel de enseñanza media del Centro de Educación Integrada de Adultos de la Comuna San Ramón

Medina, José Manuel; Toledo Pérez, Daniela; Celis Sepúlveda, Daniela; Martínez Reyes, María José Caracterización de prácticas educativas inclusivas en modalidad regular de educación para personas jóvenes y adultos. Un estudio de caso en el segundo nivel de enseñanza media del Centro de Educación Integrada de Adultos de la Comuna San Ramón

Revista Educación las Américas, vol. 8, 2019

Universidad de Las Américas, Chile

DOI: https://doi.org/10.35811/rea.v8i0.5

Esta obra está bajo una Licencia Creative Commons Atribución-SinDerivar 4.0 Internacional. 


\section{Caracterización de prácticas educativas inclusivas en modalidad regular de educación para personas jóvenes y adultos. Un estudio de caso en el segundo nivel de enseñanza media del Centro de Educación Integrada de Adultos de la Comuna San Ramón}

Characterization of inclusive educational practices in regular mode of education for young people and adults. A case study in 10th grade of night school at Centro de Educación Integrada de Adultos in San Ramón

José Manuel Medina

DOI: https://doi.org/10.35811/rea.v8i0.5

Universidad de Las Américas, Chile, Chile

jmedina@udla.cl

(DD http://orcid.org/0000-0002-1913-977X

Daniela Toledo Pérez

Universidad de Las Américas, Chile

dtoledo@udla.cl

Daniela Celis Sepúlveda

Universidad de Las Américas, Chile

daniela.celis.sepulveda@gmail.com

Maria José Martinez Reyes

Universidad de Las Américas, Chile

martinezreyes.mariajose@gmail.com

Recepción: 11 Agosto 2019

Aprobación: 11 Septiembre 2019

\section{ReSUMEN:}

La educación para adultos en Chile ha atravesado varios cambios en las últimas décadas, desde su política hasta su estructura y con ello también las prácticas educativas, en un intento por garantizar un estándar de calidad educativa y constituirse como un espacio efectivo de inclusión para aquellas personas que buscar culminar sus estudios primarios o secundarios. La presente investigación, mediante un enfoque cualitativo orientado desde el estudio de caso, tuvo como propósito central caracterizar y analizar las prácticas educativas inclusivas implementadas por profesionales de la educación especial en un Centro de Educación Integrada de Adultos en la modalidad regular en los niveles de $3^{\circ}$ y $4^{\circ}$ año de enseñanza media. A través de entrevistas y focus group, los hallazgos subrayan la debilidad en el dominio de la normativa y protocolos que permitan garantizar procesos educativos inclusivos. No obstante, los profesores direccionan sus esfuerzos hacia la flexibilización de la enseñanza y la contextualización de los aprendizajes, acciones pedagógicas que le permiten asegurar espacios educativos inclusivos, visibles y altamente valorado desde la percepción del estudiante pues favorece un aprendizaje significativo y funcional.

Palabras ClaVe: prácticas inclusivas, educación para adultos, inclusión, metodologías, aprendizaje.

\section{Abstract:}

Adult education in Chile has undergone several changes in the recent decades, from its policies to its structure affecting its educational practices. This was an attempt to guarantee an educational quality standard and become an effective space for inclusion for those people who are seeking to complete their primary or secondary studies. This investigation uses a qualitative approach in a case study. Its central purpose was to characterize and analyze the inclusive educational practices implemented by special education professionals in an Integrated Adult Education Center in the regular modality at levels of 11th and 12th grade. The findings of interviews and focus groups underscore the weakness in the domain of regulations and protocols that allow to guarantee inclusive educational processes. However, teachers direct their efforts towards the flexibility of teaching and the contextualization of learning, pedagogical actions that allow them to ensure inclusive, visible and highly valued educational spaces from the student's perception as it favors meaningful and functional learning.

KEYWORDS: inclusive practices, adult education, inclusion, methodologies, learning. 


\section{ANTECEDENTES TEÓRICOS Y EMPÍRICOS SOBRE LA EDUCACIÓN PARA ADULTOS EN CHILE}

En Chile la educación ha transcurrido por considerables cambios en la historia a nivel internacional y nacional, afectando a todos los niveles y formas en las que la educación se despliega. La educación para adultos no ha sido la excepción ni ha quedado exenta de este cambio, ya que se encuentra delimitada por la Declaración Universal de los Derechos Humanos que, en su artículo $\mathrm{N}^{\circ} 26$, declara que toda persona tiene derecho a la educación y que esta debe ser obligatoria, además de gratuita, al menos en lo concerniente a la instrucción elemental y fundamental (UNESCO, 1948). Lo anterior se constituye como un hito a nivel internación que emplaza a los distintos sistemas educativos a asegurar una estructura que respete y garantice lo articulado por esta declaración de derechos. A partir de aquí, Chile comenzó a generar cambios en su educación, con la finalidad de asegurar el derecho, gratuidad, obligatoriedad y calidad en la educación y la educación para adultos no fue la excepción, el país centro su atención en esta modalidad. Uno de los hitos que marca esta modalidad es la Ley de Instrucción Primaria Obligatoria, promulgada en el año 1920, la cual establece que la educación primaria que se imparta bajo la dirección del Estado debe ser gratuita. (Ley 3.654, 1920). Además, esta Ley reconoce y clasifica las escuelas para adultos, dando un importante paso en la disminución de la tasa de analfabetismo de la época. Es así, como Chile en las distintas conferencias y declaraciones internacionales ha realizado avances en materia educativa a nivel nacional, reconociendo la importancia del ser humano de recibir una educación digna y de calidad.

A partir de esto, el Ministerio de Educación se ha encargado de fomentar a la ciudadanía completar la enseñanza obligatoria, poniendo a disposición diversas modalidades, para acceder y continuar la escolaridad a través de horarios flexibles. Entre esas modalidades se encuentra la Educación para Personas Jóvenes y Adultas (en adelante EPJA), modalidad regular de educación de adultos dirigida a las personas jóvenes y adultas que desean iniciar o completar sus estudios, sean estos de Enseñanza Básica, Media Humanístico-Científica o Técnico-Profesional (MINEDUC, 2009). Además, quienes deseen incorporarse a Enseñanza Básica deben tener como mínimo 15 años y para ingresar a Educación Media se requiere como mínimo 17 años, cumplidos, a más tardar, al 30 de junio del año lectivo al que la persona desea incorporarse. Con respecto a lo anterior, en Chile existen aproximadamente 813 establecimientos que disponen de esta modalidad en el país.

Según el Ministerio de Educación (2009) respecto a EPJA, la Modalidad Regular de Educación de Adultos, tanto en básica como en media Humanístico Científica y Técnico Profesional, se imparte en establecimientos educacionales que cuentan con reconocimiento oficial del Ministerio de Educación, por lo tanto, los certificados que entregan a sus estudiantes les permiten continuar estudios en la educación superior: Centros de Formación Técnica, Institutos Profesionales o Universidades. En relación a lo anterior, se encuentran centros que imparten este tipo de enseñanza los Centros de Educación Integrada de Adultos (en adelante CEIA), establecimientos que funcionan como Tercera Jornada (en adelante TJ), unidades militares y penitenciarias que les permiten recibir escolaridad.

El CEIA de la comuna San Ramón es el establecimiento objeto de esta investigación. El Plan Anual de Educación Municipal (PADEM) de la comuna (Ilustre Municipalidad de San Ramón, 2017) indica que 515 estudiantes se matricularon en el año 2015. Además, ese mismo año se promovieron 395 estudiantes. A partir de esta información es importante conocer las prácticas educativas que implementa el centro para dar respuesta a las demandas de todos/as sus estudiantes.

Se puede visualizar a grandes rasgos la panorámica nacional e internacional de la educación para adultos. Sin embargo, es relevante entender el concepto de adulto. Jurídicamente, un adulto es todo aquel que ha cumplido la mayoría de edad o 18 años, tal como lo expresa el Código Civil Chileno en su Artículo $\mathrm{N}^{\circ}$ 26:

Llámase infante o niño todo el que no ha cumplido siete años; impúber, el varón que no ha cumplido catorce años y la mujer que no ha cumplido doce; adulto, el que ha dejado de ser impúber; mayor de edad, o simplemente mayor, el que ha cumplido dieciocho años; y menor de edad, o simplemente menor, el que no ha llegado a cumplirlos (Ley 19.221, 1993, párr.2). 
Cronológicamente, según el ciclo vital humano, Papalia y Martorell (2017) establecen que la etapa adulta se subdivide en cuatro, estas son: Adultez emergente, Adultez Temprana, Adultez Media y Adultez Tardía. El comienzo de la edad adulta es de 18 años en adelante, aunque, por otro lado, y para efectos de análisis de estadísticas internacionales, se considera que: "La definición internacional de adulto en las estadísticas sobre alfabetismo entre la población adulta, o su contraparte el analfabetismo, se asocia con la edad; se considera que la etapa de adulto se inicia a los quince años" (King, 1998).

Pasando a otro punto, Chile está dispuesto a velar por los derechos, políticas y decretos en la educación e inclusión, las cuales han manifestado un enfoque inclusivo que asegure el acceso y la calidad de la educación. El índice de inclusión entrega importantes aportes a la educación inclusiva, constituyéndose como un instrumento que permite guiar los procesos y las prácticas inclusivas para llevar cabo la inclusión a cabalidad hacia todos los estudiantes (Booth y Ainscow, 2000). La importancia de las prácticas inclusivas es que además de reflejar la cultura y las políticas, contribuye a una educación inclusiva, con lo que permite brindar oportunidades reales de participación en la comunidad a todos/as sus estudiantes.

\section{JUSTIFICACIÓN E IMPORTANCIA DEL PROBLEMA DE ESTUDIO}

Actualmente, la educación es un aspecto fundamental en el desarrollo humano pues permite generar reales oportunidades en la sociedad. El Estado ha realizado diversas políticas y reformas para asegurar la educación de todos los ciudadanos, asumiendo su responsabilidad al momento de garantizar el acceso, permanencia y progreso en la educación, sin importar el nivel socioeconómico, orígenes, sexo o edad; dicho compromiso se ha operacionalizado en el sistema educativo chileno a través de la modalidad conocida como "Educación de Personas Jóvenes y Adultas”, la cual acoge a estudiantes desde los 15 años de edad en adelante, que desean terminar sus estudios postergados por alguna razón. No obstante, esta modalidad educativa pareciera no ser considerada al momento de plantear nuevas políticas educativas o al generar espacios de discusión frente a la contingencia educativa nacional actual. Tomando lo anterior, nuestra investigación se vuelve útil, puesto que busca dar a conocer las prácticas educativas inclusivas que implementa el CEIA en la comuna de San Ramón, con lo que permite ampliar la mirada profesional de la Educación Especial para dar paso a nuevos campos de investigación enriquecedores en la formación de tantos Educadores y Educadoras Diferenciales, pues en las universidades se analiza el marco curricular o las bases curriculares de la educación diurna regular, la pedagogía y la didáctica se orienta hacia el aprendizaje de niños y adolescentes y poco se sabe sobre los aportes de pedagogos en etapas de la edad adulta y las metodologías más permanentes para enseñar (Letelier, 2007).

Tomando los aportes de Letelier (2007), es fundamental señalar que la investigación propuesta en el campo de la educación para adultos genera un conocimiento importante y valioso respecto a las prácticas que implementa el centro educativo. Además, social y profesionalmente es un desafío indagar en las prácticas educativas que implementa el establecimiento, pues fomentan la inclusión, logran promover a estudiantes jóvenes y adultos que llegan al establecimiento como su última opción de poder concluir sus estudios, y forman así, un estudiantado diverso en cuanto a intereses, edades y motivaciones.

En suma, es oportuna la indagación científica en establecimientos educacionales que promueven espacios de desarrollo, ya que permite que jóvenes y adultos culminen sus estudios. Específicamente, es relevante interrogar sobre las prácticas educativas de estos centros, pues permite contribuir activamente a la inclusión educativa de todas y todos los estudiantes sin distinción de sexo, origen ni mucho menos de edad.

Finalmente, con foco sobre la necesidad que emerge desde la literatura y evidencia empírica que fundamenta la presente investigación, es factible preguntarse entonces: ¿Cuáles son las normativas vigentes que regulan el CEIA de la Comuna de San Ramón para implementar prácticas inclusivas en el segundo nivel, correspondiente a $3^{\circ}$ y $4^{\circ}$ medio de la jornada vespertina?, y a partir de lo anterior consultar ¿Cuáles son las metodologías de enseñanza que utilizan estos docentes de este CEIA en particular? De igual modo es 
necesario preguntarse ¿Cuál es la percepción de los estudiantes de este nivel de la jornada vespertina respecto a las prácticas educativas inclusivas que implementa el CEIA de la comuna de San Ramón? Lo que, en conjunto, nos lleva finalmente a preguntar: ¿Cómo son las prácticas educativas inclusivas que implementa el Centro de Educación Integrada de Adultos de la comuna de San Ramón en los estudiantes que pertenecen al segundo nivel, correspondiente a $3^{\circ}$ y $4^{\circ}$ medio de la jornada vespertina?

\section{OBJETIVOS DE LA INVESTIGACIÓN}

Por una parte, el objetivo general de esta investigación fue caracterizar las prácticas educativas inclusivas implementadas en el Centro de Educación Integrada de Adultos (CEIA) de la comuna de San Ramón en los estudiantes que pertenecen al segundo nivel, correspondiente a $3^{\circ}$ y $4^{\circ}$ medio de la jornada vespertina.

Por otra parte, los objetivos específicos, fueron tres

- Identificar la normativa que utiliza el CEIA de la comuna de San Ramón para implementar prácticas educativas inclusivas en los estudiantes que pertenecen al segundo nivel, correspondiente a $3^{\circ}$ y $4^{\circ}$ medio de la jornada vespertina.

Describir las metodologías de enseñanza que utilizan los docentes del CEIA de la comuna de San Ramón con los estudiantes que pertenecen al segundo nivel, correspondiente a $3^{\circ}$ y $4^{\circ}$ medio de la jornada vespertina.

Determinar y analizar la percepción de los estudiantes del segundo nivel, correspondiente a $3^{\circ} \mathrm{y}$ $4^{\circ}$ medio de la jornada vespertina, en relación a las prácticas educativas inclusivas que implementa el Centro de Educación Integrada de Adultos (CEIA) de la comuna de San Ramón.

\section{Metodología}

Nuestra investigación está basada en el enfoque cualitativo, entendiendo que este enfoque provee información acerca del lado humano de los hechos sociales, enfocándose, desde una perspectiva subjetivista, al estudio de comportamientos, creencias, opiniones, emociones, actitud, valores, sentimientos y la relación entre las personas. Sumado a ello, Galeano (2004) señala que la investigación bajo el enfoque cualitativo contiene sus cimientos en una interrogante planteada por el investigador hasta llegar a una idea inicial, la cual se va transformando hasta la conformación de un objeto idóneo de ser investigado (Galeano, 2004). Además, esta investigación se adscribe al paradigma interpretativo puesto que, según Bisquerra (2009):

Desde este enfoque, el estudio de la realidad educativa parte de su consideración en tanto que una construcción social resultante de las interpretaciones subjetivas y los significados que le otorgan las personas que lo protagonizan. Por lo tanto, los hechos no se agotan en las manifestaciones objetivas de los fenómenos ni pueden ser entendidos por referencia a explicaciones causales (p. 74).

Asimismo, este paradigma asume, desde un punto de vista ontológico, que la realidad objetiva, como tal, no existe -a diferencia de la concepción positivista- sino que es una construcción social, resultado de la interacción humana.

Para efectos de la presente investigación, cuyo objetivo principal es caracterizar las prácticas educativas en el segundo nivel, correspondiente a $3^{\circ}$ y $4^{\circ}$ medio del Centro de Educación Integrada de Adultos de la Comuna de San Ramón, el enfoque y paradigma escogido permiten comprender la realidad de ese contexto en relación a las prácticas educativas inclusivas, como se instalan, despliegan y son comprendidas por actores claves, esto a partir de elementos normativos sistematizados estructuralmente y la interpretación de las percepciones de estos actores claves. 


\subsection{Fundamentación y descripción del diseño}

Tal como se menciona en el título de nuestra investigación, este estudio se basa en el diseño del estudio de caso. Para entender este concepto podemos constatar variadas definiciones, entre las cuales se destacan:

El estudio de caso es una estrategia de investigación cualitativa que busca documentar, interpretar y valorar, en el contexto de su desarrollo, la particularidad y complejidad de un objeto de estudio que es concreto, contemporáneo y no controlable por el investigador. (Pérez, 2016)

Por su parte, este diseño se define como:

Una investigación empírica que estudia un fenómeno contemporáneo dentro de su contexto de la vida real, especialmente cuando los límites entre el fenómeno y su contexto no son claramente evidentes. (...) Una investigación de estudio de caso trata exitosamente con una situación técnicamente distintiva en la cual hay muchas más variables de interés que datos observacionales; y, como resultado, se basa en múltiples fuentes de evidencia, con datos que deben converger en un estilo de triangulación; y, también como resultado, se beneficia del desarrollo previo de proposiciones teóricas que guían la recolección y el análisis de datos. (Yacuzzi, 2005)

El estudio de caso comprende dos características fundamentales: es una técnica cualitativa y requiere la triangulación de datos, todo esto, con el claro objetivo de contrastar y analizar la información para lograr reconstruir la realidad u objeto que se está investigando.

Es importante destacar que existen variadas formas de clasificar los estudios de caso, sin embargo, según el objetivo fundamental, se encuentran tres tipos (Stake, 1998) denominados estudio intrínseco, estudio instrumental y estudio de caso colectivo. Tomando en consideración estos tres tipos de estudio de caso, es necesario establecer que nuestro estudio de caso es de tipo intrínseco, puesto que es menester para nuestra investigación conocer en detalle la realidad elegida como objeto de estudio, mediante una descripción detallada de las prácticas inclusivas que allí ocurren. Tal como señala Stake (1998), es preciso tener presente que, el hecho de descubrir relaciones, indagar en los temas y sumar datos categóricos nos ayudará, pero estos fines están subordinados a la comprensión del caso.

Finalmente, este diseño nos permite comprender y conocer de manera detallada y profunda todas las particularidades que determinan nuestra investigación, a partir de una situación concreta y real, admitiendo una diversa utilización de fuentes de información que nos lleve a la caracterización del fenómeno investigativo que queremos abordar.

\subsection{Población y Muestra}

La investigación se realizó en el Centro de Educación Integrada de Adultos (CEIA) de La Comuna San Ramón, en la Región Metropolitana. De acuerdo a los datos extraídos del Proyecto Educativo Institucional (PEI) 2017, el nombre del establecimiento es Escuela Especial de Adultos, su RBD es 9631-9, de dependencia Municipal, por lo que el sostenedor es la Ilustre Municipalidad de San Ramón. Los niveles de enseñanza que atiende el centro son: Educación Básica Adultos sin oficios, Educación Básica Adultos con Oficio y Educación Media Científico- Humanista Adulto. Según lo señalado en el PEI (2017), el equipo directivo está compuesto por un director y dos inspectores generales.

Además, el establecimiento cuenta con tres horarios en los cuales imparten educación para personas jóvenes y adultas, los horarios son: jornada de mañana, jornada de tarde y jornada vespertina.

La muestra utilizada en esta investigación es de tipo no probabilística, ya que es una muestra intencionada y seleccionada en función a su accesibilidad o a criterio personal e intencional del investigador (Hernández, Fernández y Baptista, 2010). La muestra seleccionada correspondió a un subgrupo de la población objetivo, en este caso a informantes pertenecientes al Centro de Educación Integrada de Adultos de la Comuna 
San Ramón, estos son el equipo directivo, profesores de distintas asignaturas, estudiantes pertenecientes al segundo nivel " $D$ ", correspondiente a $3^{\circ}$ y $4^{\circ}$ año medio y una muestra representativa del equipo multidisciplinario que trabaja en torno al curso mencionado. En la tabla 1, se expone en detalle la composición de la muestra.

TABLA.1

Selección de la Muestra

\begin{tabular}{|l|l|}
\hline Informante & $\mathrm{n}=$ \\
\hline Director & 1 \\
\hline $\begin{array}{l}\text { Jefa de Unidad Técnico } \\
\text { Pedagógica }\end{array}$ & 1 \\
\hline $\begin{array}{l}\text { Profesora de Educación } \\
\text { Diferencial }\end{array}$ & 1 \\
\hline Psicopedagoga & 1 \\
\hline Profesor de Educación Física & 1 \\
\hline Profesora de Inglés & 1 \\
\hline Profesor de Historia & 1 \\
\hline Profesor de Matemáticas & 1 \\
\hline Profesora de Filosofía & 1 \\
\hline Estudiantes & 6 \\
\hline Total & 15 \\
\hline
\end{tabular}

Elaboración propia.

\subsection{Fundamentación y descripción de técnicas e instrumentos}

Para la recopilación de información, es fundamental seleccionar técnicas e instrumentos idóneos que permitan una recogida de datos pertinente y acabada además de lograr proporcionar información específica y relevante para nuestro estudio. Desde ese punto de vista y considerando el enfoque cualitativo de nuestra investigación,

Las técnicas de recogida de información se refieren a la búsqueda de la palabra, y por lo tanto requieren una actitud de escucha. Dentro de este encuadre metodológico existen varias técnicas a utilizar, [...] la entrevista en profundidad semiestructurada, el grupo focal o de discusión y la observación participante. (Huenchuan y Paredes, 2007)

Desde las técnicas e instrumentos que se declaran en la cita anterior, el grupo focal o de discusión y la entrevista semi-estructurada son las más idóneas para recolectar información en nuestra investigación.

Respecto a los grupos focales o de discusión, se define como "una técnica que propicia la exploración de un tema a partir de la interacción entre los participantes." (Kornblit, 2007). Además, se establece que "el grupo focal es particularmente útil para explorar los conocimientos, las prácticas y las opiniones, no sólo en el sentido de examinar lo que la gente piensa sino también cómo y por qué piensa como piensa (Kornblit, 2007).

Respecto a la realización de esta técnica, se indica que "los temas tratados en el grupo son dirigidos o conducidos por un moderador (...), quien se ocupa de plantear los tópicos, acotar y ordenar las intervenciones de los participantes y marcar el inicio y final del encuentro" (Huenchuan y Paredes, 2007, p.36).

Tomando como referencia lo anteriormente señalado, resulta pertinente aplicar esta técnica a los estudiantes del segundo nivel correspondiente a $3^{\circ}$ y $4^{\circ}$ medio del Centro de Educación Integrada de Adultos, para así contrastar las diferencias de opiniones que pudieran existir en ese grupo delimitado respecto a las prácticas educativas inclusivas implementadas por el establecimiento.

La segunda técnica elegida corresponde a la entrevista semi- estructurada, instrumento que: 
Pretende recoger o corroborar una información determinada sobre ciertos aspectos muy concretos, a través de algunas preguntas precisas, a la vez que le interesa recoger información más abierta. [...] Se suele utilizar cuando ya se va centrando el problema de la investigación y se van delimitando ciertas dimensiones, para recoger información más precisa, contrastarla con los informantes, corroborarla y ampliarla (Villegas, 2011).

Para el tipo de investigación que va enfocado, este instrumento logra conseguir mayor información, ya que, a medida que se realiza la entrevista, pueden surgir nuevas interrogantes que permitan indagar de manera más precisa en la investigación. En otras palabras, este instrumento se vuelve más flexible y dinámico. Fue elegido por esta razón, ya que permite ahondar de manera eficiente en la caracterización de las prácticas inclusivas impartidas por el Centro de Educación Integrada de Adultos de San Ramón en el segundo nivel correspondiente a $3^{\circ}$ y $4^{\circ}$ medio. Es por lo anterior, que resulta adecuado aplicar esta técnica al equipo directivo del establecimiento mencionado y al equipo multidisciplinario que trabaja con el $2^{\circ}$ nivel del centro educativo, permitiendo de esta manera, indagar exhaustivamente en la temática antes señalada.

\subsection{Criterios de rigor y éticos en la investigación cualitativa}

El rigor es un concepto transversal en el desarrollo de un proyecto de investigación y permite valorar la aplicación escrupulosa y científica de los métodos de investigación, y de las técnicas de análisis para la obtención y el procesamiento de los datos (Noreña, ALcaraz, Rojas y Rebolledo, 2012).

Para la presente investigación los criterios de rigor son los que indicamos en la tabla 2. 
TABLA 2

Criterios de rigor

\begin{tabular}{|c|c|c|}
\hline Criterio & $\begin{array}{l}\text { Característica del } \\
\text { Criterio }\end{array}$ & Procedimiento \\
\hline $\begin{array}{l}\text { Credibilidad } \\
\text { Valor de la } \\
\text { verdad / } \\
\text { Autenticidad }\end{array}$ & $\begin{array}{l}\text { Aproximación de } \\
\text { los resultados de } \\
\text { una investigación } \\
\text { frente al fenómeno } \\
\text { observado. }\end{array}$ & $\begin{array}{l}\text { Triangulación /complementariedad } \\
\text { de los datos de la investigación, } \\
\text { tanto con su fase cuantitativa } \\
\text { como con el estado del arte en } \\
\text { este campo de investigación. } \\
\text { Los resultados son reconocidos } \\
\text { como "Verdaderos" por } \\
\text { los participantes. }\end{array}$ \\
\hline $\begin{array}{l}\text { Transferibilidad } \\
\text { Aplicabilidad }\end{array}$ & $\begin{array}{l}\text { Los resultados } \\
\text { derivados de la } \\
\text { investigación } \\
\text { cualitativa no son } \\
\text { generalizables sino } \\
\text { transferibles. }\end{array}$ & $\begin{array}{l}\text { Descripción detallada del } \\
\text { contexto y de los participantes. } \\
\text { Recogida exhaustiva de datos. }\end{array}$ \\
\hline $\begin{array}{l}\text { Consistencia } \\
\text { Dependencia/ } \\
\text { Replicabilidad }\end{array}$ & $\begin{array}{l}\text { La complejidad de } \\
\text { la investigación } \\
\text { cualitativa dificulta } \\
\text { la estabilidad de los } \\
\text { datos. Tampoco es } \\
\text { posible la } \\
\text { replicabilidad } \\
\text { exacta del estudio. }\end{array}$ & $\begin{array}{l}\text { Descripción detallada del } \\
\text { proceso de recogida, análisis e } \\
\text { interpretación de los datos. } \\
\text { Reflexividad del investigador. }\end{array}$ \\
\hline $\begin{array}{l}\text { Confirmabilidad } \\
\text { o Reflexividad } \\
\text { Neutralidad } \\
\text { / Objetividad }\end{array}$ & $\begin{array}{l}\text { Los resultados de la } \\
\text { investigación deben } \\
\text { garantizar la } \\
\text { veracidad de las } \\
\text { descripciones } \\
\text { realizadas por los } \\
\text { participantes. }\end{array}$ & $\begin{array}{l}\text { Transcripciones } \\
\text { textuales de la } \\
\text { información recogida. } \\
\text { Contrastación de los } \\
\text { resultados con la literatura } \\
\text { existente. Revisión de hallazgos } \\
\text { por otros investigadores. }\end{array}$ \\
\hline Relevancia & $\begin{array}{l}\text { Permite evaluar el } \\
\text { logro de los } \\
\text { objetivos } \\
\text { planteados y saber } \\
\text { si se obtuvo un } \\
\text { mejor conocimiento } \\
\text { del fenómeno de } \\
\text { estudio }\end{array}$ & $\begin{array}{l}\text { Configuración de nuevos } \\
\text { planteamientos teóricos o } \\
\text { conceptuales. · Comprensión } \\
\text { amplia del fenómeno. } \\
\text { Correspondencia entre la } \\
\text { justificación y los resultados } \\
\text { obtenidos. }\end{array}$ \\
\hline
\end{tabular}

Elaboración propia.

Por su parte, la ética cualitativa busca el acercamiento a la realidad del ser humano de manera holística y con mínima intrusión, otorgando libertad a los participantes y considerándolos sujetos, no objetos de estudio (Noreña y otros, 2012).

Los criterios éticos que a continuación se detallan en la tabla 3 tienen por objetivo resguardar la integralidad del sujeto como persona, participante e informante esencial para la investigación. 
TABLA 3

Criterios éticos

\begin{tabular}{|c|c|}
\hline Criterios & Características éticas del criterio \\
\hline $\begin{array}{l}\text { Consentimiento } \\
\text { informado }\end{array}$ & $\begin{array}{l}\text { Los participantes deben estar de acuerdo con ser } \\
\text { informantes y conocer sus derechos y } \\
\text { responsabilidades. Para ello, los participantes } \\
\text { completarán una carta/ formulario con sus datos } \\
\text { personales y firma correspondiente, previa lectura } \\
\text { de los propósitos y procedimientos a seguir. }\end{array}$ \\
\hline Confidencialidad & $\begin{array}{l}\text { Asegurar la protección de la identidad de las } \\
\text { personas que participan como informantes de la } \\
\text { investigación. }\end{array}$ \\
\hline $\begin{array}{l}\text { Manejo de } \\
\text { riesgos }\end{array}$ & $\begin{array}{l}\text { Para este requisito esta investigación tendrá } \\
\text { presente los principios relacionados con la no } \\
\text { maleficencia y beneficencia establecidos para } \\
\text { hacer investigación con seres humanos. }\end{array}$ \\
\hline $\begin{array}{l}\text { Entrevista } \\
\text { individual o } \\
\text { grupal (Focus } \\
\text { group) }\end{array}$ & $\begin{array}{l}\text { Se trata de una interacción social donde no se } \\
\text { deben provocar actitudes que condicionen las } \\
\text { respuestas de los participantes (Noreña y otros., } \\
\text { 2012). }\end{array}$ \\
\hline $\begin{array}{l}\text { Grabaciones } \\
\text { de audio o video }\end{array}$ & $\begin{array}{l}\text { Dicho material deberá resguardarse en archivos } \\
\text { confidenciales y el investigador necesita ser } \\
\text { cauteloso anteponiendo la confidencialidad, el } \\
\text { respeto y el anonimato de los participantes. }\end{array}$ \\
\hline
\end{tabular}

Elaboración propia.

\section{Resultados}

Las entrevistas semiestructuradas fueron aplicadas al equipo directivo, conformado por director y Jefa de Unidad Técnica Pedagógica, y una muestra representativa del equipo multidisciplinario que trabaja con el $2^{\circ}$ nivel "D", constituido por profesores de asignatura, psicopedagoga y profesora de educación diferencial. El focus group fue aplicado a los estudiantes del segundo nivel "D”. Con toda la información recopilada se establecieron categorías y subcategorías que dan información clave a nuestra investigación, como se aprecia en el esquema 1. 


\begin{tabular}{|c|c|c|}
\hline $\begin{array}{l}\text { Objetivo Específico No I: } \\
\text { Identificar la normativa que utiliza el CEIA de la } \\
\text { comuna de San Ramón para implementar prácticas } \\
\text { educativas inclusivas en los estudiantes que pertenecen } \\
\text { al segundo nivel, correspondiente a } 3^{\circ} \text { y } 4^{\circ} \text { medio de la } \\
\text { jornada vespertina. }\end{array}$ & $\begin{array}{l}\text { Categoría } \\
\text { № I: } \\
\text { Normativa } \\
\text { vigente para } \\
\text { implementar } \\
\text { prácticas } \\
\text { inclusivas. }\end{array}$ & $\begin{array}{l}\text { Subcategoría } \mathrm{N}^{\circ} \mathbf{1 . 2} \text { : } \\
\text { Aplicación de normativas } \\
\text { de Inclusión educativa. }\end{array}$ \\
\hline \multirow[t]{2}{*}{$\begin{array}{l}\text { Objetivo Específico № 2: } \\
\text { Describir las metodologías de enseñanza que utilizan } \\
\text { los docentes del CEIA de la comuna de San Ramón } \\
\text { con los estudiantes que pertenecen al segundo nivel, } \\
\text { correspondiente a } 3^{\circ} \text { y } 4^{\circ} \text { medio de la jornada } \\
\text { vespertina. }\end{array}$} & \multirow[t]{2}{*}{$\begin{array}{l}\text { Categoría } \\
\text { № 2: } \\
\text { Metodología } \\
\text { de enseñanza } \\
\text { para adultos. }\end{array}$} & $\begin{array}{l}\text { Subcategoría } \mathrm{N}^{\circ} \text { 2.I: } \\
\text { Conocimiento } \\
\text { aplicación de } \\
\text { metodologías de } \\
\text { enseñanza para adultos. }\end{array}$ \\
\hline & & $\begin{array}{l}\text { Subcategoría } \mathbf{N}^{\circ} \mathbf{2 . 2} \\
\text { Estrategias de aprendizaje } \\
\text { para el adulto. }\end{array}$ \\
\hline \multirow{3}{*}{$\begin{array}{l}\text { Objetivo Especifico Ne } 3 \text { : } \\
\text { Determinar y analizar la percepción de los estudiantes del } \\
\text { segundonivel, correspondiente } a 3^{\circ} \text { y } 4^{\circ} \text { medio de la jornada } \\
\text { vespertina en relación a las prácticas educativas indusivas } \\
\text { que implementa el Centro de Educación Integrada de } \\
\text { Adultos (CEIA) de la comuna de San Ramón. }\end{array}$} & \multirow{3}{*}{$\begin{array}{l}\text { Categoría } \\
\text { № 3: } \\
\text { Percepción de } \\
\text { los estudiantes } \\
\text { del } 2^{\circ} \text { nivel "D" } \\
\text { sobre las } \\
\text { prácticas } \\
\text { educativas } \\
\text { inclusivas. }\end{array}$} & $\begin{array}{l}\text { Subcategoría } \mathrm{N}^{\circ} \text { 3.I: } \\
\text { Conocimiento de inclusión } \\
\text { educativa. }\end{array}$ \\
\hline & & $\begin{array}{l}\text { Subcategoría } \mathrm{N}^{\circ} 3.2 \text { : } \\
\text { Experiencias de prácticas } \\
\text { inclusivas. }\end{array}$ \\
\hline & & $\begin{array}{l}\text { Subcategoría } \mathrm{N}^{\circ} 3 \cdot 3 \text { : } \\
\text { Experiencias de aprendizaje } \\
\text { personal. }\end{array}$ \\
\hline
\end{tabular}

ESQUEMA 1

Elaboración propia.

Luego de presentadas las categorías y subcategorías que conforman nuestra investigación, es fundamental dar a conocer a los colaboradores que participaron en el presente estudio. En la tabla 4 se detalla la codificación de profesionales y estudiantes del Centro de Educación Integrada de Adultos de la comuna de San Ramón que contribuyeron a la investigación.

TABLA 4

Codificación de colaboradores

\begin{tabular}{|l|l|}
\hline Colaborador & CODIGO \\
\hline Director & DIR \\
\hline $\begin{array}{l}\text { Jefa de Unidad Técnico } \\
\text { Pedagógica }\end{array}$ & UTP \\
\hline $\begin{array}{l}\text { Profesora de Educación } \\
\text { Diferencial }\end{array}$ & PED \\
\hline Psicopedagoga & PSP \\
\hline Profesor de Educación Física & PEF \\
\hline Profesora de Inglés & PEI \\
\hline Profesor de Historia & PEH \\
\hline Profesor de Matemáticas & PEM \\
\hline Profesora de Filosofía & PFI \\
\hline Estudiante $N^{\circ} 1$ & ES1 \\
\hline Estudiante $N^{\circ} 2$ & ES2 \\
\hline Estudiante $N^{\circ} 3$ & ES3 \\
\hline Estudiante $N^{\circ} 4$ & ES4 \\
\hline Estudiante $N^{\circ} 5$ & ES5 \\
\hline Estudiante $N^{\circ} 6$ & ES6 \\
\hline
\end{tabular}

Elaboración propia 
A continuación, se exponen las categorías y subcategorías en la tabla 5, tabla 6 y tabla 7 .

TABLA 5

Análisis Categoría $\mathrm{N}^{\circ}$ 1: Normativa vigente para implementar prácticas inclusivas

\begin{tabular}{|c|c|}
\hline \multicolumn{2}{|l|}{$\begin{array}{l}\text { Categoria } N^{\circ} 1 \text { : Norm } \\
\text { inclusivas }\end{array}$} \\
\hline Subcategoría $N^{\circ} 1.1$ & \\
\hline bcategoría $\mathrm{N}^{\circ} 1.2$ & \\
\hline strumento/Técnica & \\
\hline \multicolumn{2}{|c|}{ Sintesis de categoría $\mathrm{N}^{\circ} 1$} \\
\hline \multicolumn{2}{|c|}{$\begin{array}{l}\text { Luego de analizadas ambas subcategorias que componen la } \\
\text { categoria de "Normativas vigentes para implementar prácticas } \\
\text { inclusivas", es posible establecer que el equipo directivo del Centro } \\
\text { de Educación Integrada de Adultos de la comuna de San Ramón, } \\
\text { conoce a modo general algunas de las normativas legales vigentes } \\
\text { que favorecen la inclusión educativa, entre las que destacan se } \\
\text { encuentra: Ley de Inclusión y Política Nacional de Convivencia } \\
\text { Escolar. Por otro lado, el equipo directivo demuestra a lo largo de la } \\
\text { entrevista que efectivamente se aplican algunas normativas legales } \\
\text { vigentes que promueven la inclusión educativa. Un ejemplo de esto } \\
\text { es que no poseen sistema de selección de estudiantes y realizan } \\
\text { flexibilización de la enseñanza, entre otros. Tomando lo anterior, es } \\
\text { posible destacar que el equipo directivo valora profundamente el } \\
\text { impacto que generan estas normativas en la convivencia escolar, } \\
\text { brindando oportunidades a todos los participantes de la comunidad. } \\
\text { No obstante, la aplicación antes mencionada, es intuitiva puesto } \\
\text { que, los entrevistados o equipo directivo poseen nociones generales } \\
\text { de las normativas vigentes al momento de la entrevista. }\end{array}$} \\
\hline
\end{tabular}

Elaboración propia. 
José Manuel Medina, et al. Caracterización de PrÁcticas educativas inclusivas en modalidad RegulaR...

TABLA 6

Análisis Categoría N²: Metodologías de enseñanza para adultos

\begin{tabular}{|l|l|}
\hline Categoria $\mathrm{N}^{\circ}$ 2: Metodologías de enseñanza para adultos \\
\hline Subcategoría $\mathrm{N}^{\circ} 2.1$ & $\begin{array}{l}\text { Conocimiento y aplicación de metodologias } \\
\text { de enseñanza para adultos. }\end{array}$ \\
\hline Subcategoría $\mathrm{N}^{\circ} 2.2$ & EStrategias de aprendizaje para el adulto. \\
\hline Instrumento/Técnica & $\begin{array}{l}\text { Entrevista / CoD Informante: PFI-PSP-PEH- } \\
\text { PEI-PED-PEF-PEM }\end{array}$ \\
\hline Síntesis de categoría $\mathrm{N}^{\circ} 2$ \\
\hline $\begin{array}{l}\text { Luego de analizadas ambas subcategorias que componen la } \\
\text { categoría de "Metodologías de enseñanza para adultos" es posible } \\
\text { establecer que, en el Centro de Educación Integrada de Adultos de la } \\
\text { comuna de San Ramón, se aplican algunos conceptos claves de la } \\
\text { metodologia de enseñanza para el adulto propuesta por Paulo Freire, } \\
\text { la cual debe estar contextualizada a la vida cotidiana de los } \\
\text { estudiantes. Los profesores y profesionales vinculados al segundo } \\
\text { nivel "D", comparten la percepción de la necesidad de vincular la } \\
\text { enseñanza que ellos transmiten con el contexto en el que los } \\
\text { estudiantes adultos se desenvuelven, logrando asi, un aprendizaje } \\
\text { funcional y significativo. Sin embargo, cabe destacar que Paulo } \\
\text { Freire menciona la importancia de la problematización en la } \\
\text { enseñanza del adulto. Ante esto, solo una profesora de la muestra } \\
\text { representativa de los profesionales declara que en su metodología } \\
\text { incorpora la problematización en su enseñanza. Otro punto } \\
\text { trascendental, dice relación con la importancia que el equipo } \\
\text { multidisciplinario atribuye al trabajo entre pares, favoreciendo un } \\
\text { aprendizaje socializado, contextualizado y vinculado a la } \\
\text { cotidianidad del estudiante. }\end{array}$ \\
\hline
\end{tabular}

Elaboración propia 
TABLA 7

Análisis Categoría $N^{\circ} 3$ : Percepción de los estudiantes $\operatorname{del} 2^{\circ}$ nivel "D" sobre las prácticas educativas inclusivas

\begin{tabular}{|c|c|}
\hline \multicolumn{2}{|c|}{$\begin{array}{l}\text { Categoría } \mathrm{N}^{\circ} 3 \text { : Percepción de los estudiantes del } 2^{\circ} \text { nivel " } \mathrm{D} \text { " sobre } \\
\text { las prácticas educativas inclusivas }\end{array}$} \\
\hline Subcategoría $\mathrm{N}^{\circ} 3.1$ & Conocimiento de inclusión educativa. \\
\hline Subcategoría $\mathrm{N}^{\circ} 3.2$ & \\
\hline Subcategoría $N^{\circ} 3.3$ & Experiencias de aprendizaje personal. \\
\hline nto/Técnica & \\
\hline \multicolumn{2}{|c|}{ Sintesis de categoria $\mathrm{N}^{\circ} 3$} \\
\hline \multicolumn{2}{|c|}{$\begin{array}{l}\text { Una vez conocidas las interpretaciones de cada subcategoría que } \\
\text { compone la categoria de "Percepción de los estudiantes del } 2^{\circ} \text { nivel } \\
\text { "D" sobre las prácticas educativas inclusivas", es posible evidenciar } \\
\text { que los estudiantes mantienen una opinión positiva respecto a las } \\
\text { prácticas que implementa el centro educativo. Asi se refleja en cada } \\
\text { una de las respuestas obtenidas en el focus group. Tomando lo } \\
\text { anterior, cuando los estudiantes son consultados respecto al concepto } \\
\text { de inclusión educativa, ellos entregan más bien un concepto ligado a } \\
\text { la integración educativa, sin embargo, logran establecer la relación } \\
\text { que posee inclusión con diversidad en la comunidad escolar. Además, } \\
\text { al momento de referirse a la existencia de prácticas donde se asegure } \\
\text { la participación de todos los estudiantes, ellos coinciden y destacan } \\
\text { las paracadémicas, la cual, es una actividad que comprende la } \\
\text { realización de una disertación, muestra gastronómica y de baile. A } \\
\text { partir de esto, es posible observar que los estudiantes valoran esta } \\
\text { práctica y explicitan que corresponde a una práctica inclusiva. } \\
\text { Finalmente, respecto a los motivos personales de retomar los estudios } \\
\text { que los estudiantes refieren, se encuentra principalmente la mejora de } \\
\text { oportunidades laborales y el cierre de una etapa de la vida } \\
\text { postergada. Ante estos dos puntos, es necesario destacar la } \\
\text { motivación intrinseca de los estudiantes jóvenes y adultos, la cual, } \\
\text { impacta positivamente en su formación académica, cumpliendo así la } \\
\text { etapa escolar. }\end{array}$} \\
\hline
\end{tabular}

Elaboración propia

\section{Conclusiones}

Esta investigación tuvo como propósito central indagar sobre las características que configuran las prácticas educativas inclusivas que implementa el CEIA de la comuna de San Ramón en un segundo nivel de la jornada vespertina, tanto desde su estructura normativa como desde la percepción de los actores involucrados.

Basados en el análisis y discusión de los datos obtenidos, podemos señalar que el centro educativo se rige bajo normativas que favorecen la inclusión educativa. Informantes claves en este estudio manifiestan que la Ley de Inclusión y la Política Nacional de Convivencia Escolar son fundamentales en el funcionamiento y prácticas que lleva a cabo el establecimiento, sin embargo, el equipo directivo no posee protocolos que regulen las prácticas que ellos ejecutan, generando así, una gestión desorganizada respecto a sus acciones educativas. Cabe señalar que, en contraste con lo anterior, el Ministerio de Educación establece la importancia de que los centros educativos reflejen en sus documentos y protocolos institucionales las prácticas que lleva a cabo para asegurar la participación de todo el estudiantado; con foco en esta ordenanza, es imperativo que cualquier establecimiento ordene sus prácticas educativas por medio de instrumentos de gestión institucional que le permitan lograr alinear lo propuesto con las acciones a implementar y vincular efectivamente a toda la comunidad educativa en torno a un mismo objetivo.

En correspondencia con lo señalado, la información recopilada permite inferir que existen nociones generales y débiles respecto a las normativas que favorecen la inclusión educativa, específicamente en torno 
a la Ley de Inclusión y la Política Nacional de Convivencia Escolar o sobre el Decreto $\mathrm{N}^{\circ} 170$ y Decreto N - 83. Esta debilidad relacionada con el manejo de normativas podría explicar, en parte, la falta de protocolos existentes para institucionalizar las prácticas que ejecutan conllevando así, a una desorganización de gestión evidente.

En relación al conocimiento de las metodologías de enseñanza que utilizan los docentes del establecimiento, los profesores mencionan que realizan constantemente flexibilización de la enseñanza realizando algunas acciones, tales como adecuaciones curriculares y variadas formas de presentación de la enseñanza. Desde los aportes teóricos y empíricos sobre la andragogía, se establece que una de las características principales de la enseñanza en el adulto es la contextualización de los aprendizajes, esto quiere decir que es necesario que los aprendizajes que adquiere el estudiante adulto se vinculen con su cotidianidad, para lograr así, un aprendizaje significativo y funcional. En correlación, los profesores y profesionales que participaron del estudio manifestaran la importancia de establecer una enseñanza basada y adaptada al contexto de los estudiantes, no obstante, en cuanto al conocimiento de metodologías de enseñanza para el adulto, se encontró que algunos profesionales poseen una confusión entre metodologías de enseñanza y estrategias de enseñanza, la cual responde a la falta de especialización disponible para la educación en adultos. Lo anterior pone de relieve desde la perspectiva social, ministerial y profesional, la necesidad de mantener vigente la discusión en torno a la educación de adultos, basados en la evidencia latente sobre la escasa información y capacitación disponible respecto a las metodologías y estrategias de enseñanza para el adulto; en razón de ello, la literatura especializada muestra que las mallas curriculares de formación profesional de profesores en Chile no se encuentran asignaturas ligadas a la enseñanza y aprendizaje del adulto, lo cual, repercute en el quehacer pedagógico al momento de abordar las demandas educativas de un estudiante adulto.

En relación a los estudiantes y a los motivos que "llaman" a retomar los estudios, estos manifiestan que uno de ellos, sino el principal, es de índole familiar, puesto que todos ellos coinciden en que su motivación principal es beneficiar directamente a sus hijos o familiares más cercanos, ya sea, con mejores oportunidades laborales o para poder apoyar en el proceso educativo de su familia. Esto permite confirmar que efectivamente existe una motivación intrínseca en el adulto por culminar sus estudios, tal como lo menciona la teoría andragógica, lo que nos permite establecer que, en este caso, el propósito principal del adulto por culminar sus estudios pretende impactar y favorecer a su núcleo familiar mediato.

Además, existe una percepción positiva de los estudiantes, que destacan la existencia de prácticas educativas inclusivas que logran asegurar la participación de todos los estudiantes. Dentro de estas prácticas los estudiantes enfatizan principalmente dos áreas: el apoyo del profesorado y una actividad transversal llamada "Paracadémicas". En relación a la percepción de los estudiantes frente al apoyo de los docentes que trabajan con el curso, manifiestan que cada profesional adapta la enseñanza tomando como base las características de ellos, facilitando el aprendizaje; indican además que la enseñanza entregada en el establecimiento es útil y necesaria para su desarrollo personal y laboral. En relación a las paracadémicas, los estudiantes mencionan que se logra articular los recursos humanos y materiales con los que dispone el establecimiento para favorecer la participación de todo el estudiantado, considerando y valorando las habilidades e intereses personales.

Finalmente, es necesario acentuar la proyección que posee este estudio, primero, como un posible insumo y/o instrumento de reflexión sobre el quehacer de este centro educativo e implementar mejoras posibles. Segundo, es primordial que este estudio forje los cimientos de nuevas investigaciones respecto a la educación de adultos, respondiendo interrogantes emanadas de los hallazgos de esa investigación y la complejidad del fenómeno estudiado: ¿Cómo impacta la escasa formación de profesores en cuanto al aprendizaje del adulto en su quehacer profesional? ¿Cuáles son las competencias que deben tener una profesora o un profesor de educación diferencial para abordar las necesidades educativas de un estudiante adulto? Así mismo, esta investigación invita a discutir y profundizar sobre la formación de futuras y futuros profesores de Educación Diferencial, para lograr una mirada amplia hacia nuevos campos formativos, a través de la búsqueda constante e incansable hacia la inclusión educativa. 


\section{REFERENCIAS}

Bisquerra, R. (2009). Metodología de la investigación educativa. Madrid: Editorial La Muralla.

Booth, T. \& Ainscow, M. (2000). Índice de Inclusión. Santiago de Chile: orealc/unesco. Recuperado de http:// www.eenet.org.uk/resources/docs/Index\%20Spanish\%20South\%20America\%20.pdf.

Centro de Educación Integrada de Adultos San Ramón. (2017). Proyecto Educativo Institucional Escuela Especial de Adultos. Santiago, Chile: Programa Interdisciplinario de Investigaciones en Educación - PIIE.

Galeano, M. (2004).Diseño deproyectos en la Investigación Cualitativa. Medellín: Fondo Editorial Universidad EAFIT.

Hernández, R.; Fernández, C. y Baptista, P. (2010). Metodología de la investigación (quinta edición). Ciudad de México: McGraw-Hill.

Huenchuan, S. y Paredes, M. (2007). Guía metodológica para la evaluación participativa de politicas y programas, en el marco de la Estrategia Regional sobre el Envejecimiento. Santiago, Chile: CEPAL.

Ilustre Municipalidad de San Ramón. (2017). Plan Anual de Desarrollo Municipal (PADEM). Recuperado de http:// transparencia.municipalidadsanramon.cl/wp-content/uploads/2016/11/P-A-D-E-M-2017.pdf.

King, L. (1998). Visiones y reflexiones: Nuevas perspectivas en la Educación de Adultos para Pueblos Indigenas. Distrito Federal, México: Editores Plaza y Valdés.

Kornblit, A. (2007). Metodología cualitativa: modelos y procedimientos de análisis. Buenos Aires: Editorial Biblos.

Letelier, M. (2007). Un nuevo marco curricular para la renovación de la educación de personas jóvenes y adultas. Santiago de Chile: MINEDUC, Programa Chile-califica.

Ley N 3.654. Diario oficial de la República de Chile, Santiago, Chile, 26 de agosto de 1920.

Ley N¹9.221. Diario oficial de la República de Chile, Santiago, Chile, 01 de junio de 1993.

Ministerio de Educación de Chile. (2009). Educación para Personas Jóvenes y Adultas. Recuperado de: https:// epja.mineduc.cl/.

Noreña, A., Alcaraz, N., Rojas, J. y Rebolledo, D. (2012). Aplicabilidad de los criterios de rigor y éticos en la investigación cualitativa. Revista Aquichan, 12(3), 263-274.

Papalia, D., \& Martorell, G. (2017). Desarrollo Humano. México, Distrito Federal: McGraw-Hill.

Pérez, O. (2016). Metodología para la elaboración de estudio de caso en Responsabilidad Social. Colombia: Universidad Externado

Stake, R. E. (1998). Investigación con estudio de casos. Madrid: Ediciones Morata.

UNESCO. (1948). Declaración Universal de los Derechos Humanos. Recuperado de http:// www.derechoshumanos.net/normativa/normas/1948-DeclaracionUniversal.htm? gclid=CjwKCAjw8O7bBRB0EiwAfbrThxhJ128v29Egfh3VOBtJl-f5uQ-WIo3U4pYmCGQUizhZb0LpuHthoCOUMQAvD_BwE.

Villegas, E. (2011). Investigación y práctica en la educación de personas adultas. Valencia: Nau Llibres.

Yacuzzi, E. (2005). El estudio de caso como metodología de investigación: Teoría, mecanismos causales, validación. Recuperado de https://www.econstor.eu/bitstream/10419/84390/1/496805126.pdf.

CC BY-ND 